1 Population Health Research Institute, St George University of London, London, UK

2 St George's University Hospitals NHS Foundation Trust, London, UK

3 Usher Institute, College of Medicine and Veterinary Medicine, University of Edinburgh, Edinburgh, UK

4 Department of Primary Care and Public Health, Imperial College London, London, UK

Correspondence to M Razai mrazai@sgul.ac.uk Cite this as: BMJ 2021;373:n1138 http://dx.doi.org/10.1136/bmi.n1138 Published: 20 May 2021

PRACTICE POINTER

\title{
Covid-19 vaccination hesitancy
}

\section{Mohammad S Razai, ${ }^{1}$ Umar A R Chaudhry, ${ }^{1}$ Katja Doerholt, ${ }^{2}$ Linda Bauld, ${ }^{3}$ Azeem Majeed ${ }^{4}$}

\section{What you need to know}

- Lack of confidence in vaccines for covid-19 poses direct and indirect threats to health, and could derail efforts to end the current pandemic

- Concerns about unknown future effects, side effects, and a lack of trust are common reasons given by people who say they are unlikely to have a covid-19 vaccine

- No single intervention is likely to be able to address vaccine hesitancy

- Consider barriers to uptake of vaccination at a population level and in groups who have lower rates of vaccine uptake

- Develop local approaches by engaging members of the community and co-producing communications and materials that meet population needs

Rollout of covid-19 vaccination is well underway, with more than 700 million doses given worldwide as of April 2021. ${ }^{1}$ Vaccination is highly effective at reducing severe illness and death from covid-19. Vaccines for covid-19 are also safe, with extremely low risks of severe adverse events. ${ }^{2-4} \mathrm{~A}$ major threat to the impact of vaccination in preventing disease and death from covid-19 is low uptake of vaccines. In this practice pointer we offer on overview of vaccine hesitancy and some approaches that clinicians and policymakers can adopt at the individual and community levels to help people make informed decisions about covid-19 vaccination.

\section{What is vaccine hesitancy?}

The World Health Organization defines vaccine hesitancy as a "delay in acceptance or refusal of safe vaccines despite availability of vaccine services." It is caused by complex, context specific factors that vary across time, place, and different vaccines, and is influenced by issues such as complacency, convenience, confidence, and sociodemographic contexts. ${ }^{6}$ Vaccine hesitancy may also be related to misinformation and conspiracy theories which are often spread online, including through social media. ${ }^{78}$ In addition, structural factors such as health inequalities, socioeconomic disadvantages, systemic racism, and barriers to access are key drivers of low confidence in vaccines and poor uptake. . $^{-11}$ The term vaccine hesitancy, although widely used, may not adequately convey these wider determinants that influence decisions to delay or refuse vaccination.

\section{How common is vaccine hesitancy?}

Vaccine hesitancy is a global problem. Surveys in 2021 report that between $50 \%$ and $60 \%$ of all respondents worldwide would be willing to receive a covid-19 vaccine, with wide variations across countries. ${ }^{1213}$ In the UK, surveys have found variation in willingness to have a vaccine between ethnic groups. The UK Household Longitudinal survey asked 12035 participants (in November 2020) "how likely or unlikely would you be to take the vaccine?” Overall only $18 \%$ of respondents were hesitant (answering unlikely or very unlikely), in contrast with high levels of hesitancy in people of Black ethnicity (72\%) followed by South Asians of Pakistani and Bangladeshi heritage (both $42 \%$ ), and mixed ethnicities (32\%), though levels of vaccine hesitancy were comparable with White people in respondents of Chinese ethnicity. ${ }^{14}$ UK data (as of 11 March 2021) show lower vaccination rates (among those eligible for vaccination) in Black African and Black Caribbean (58.8\% and 68.7\%, respectively), Bangladeshi $(72.7 \%)$, and Pakistani (74\%) ethnic groups compared with White British (91.3\%), and lower vaccination rates in people who live in more deprived areas (most deprived $87 \%$, least deprived $92.1 \%) .{ }^{15}$

Higher vaccine hesitancy is also reported among women (women $21 \%$, men $15 \%$ ), younger age groups (28\% in 25-34 years, versus $14 \%$ in 55-64 years), and in people with a lower education level (24\% in secondary school graduates; $13 \%$ in university graduates). ${ }^{14}$ These data follow a historical trend in the UK of lower uptake of pneumococcal, influenza, rotavirus, and shingles vaccines among socioeconomically disadvantaged individuals ${ }^{16-18}$ and ethnic minorities. ${ }^{11}{ }^{19}$ Similarly, a lower uptake has been observed with childhood immunisations in ethnic minority populations. ${ }^{11}$ Variation in covid-19 vaccination rates is also seen between religious groups. Vaccination rates have been lower in Muslim (72.3\%), Buddhist (78.1\%), Sikh (87\%), and Hindu $(87.1 \%)$ groups compared with Christian (91.1\%). ${ }^{15}$

Vaccine hesitancy among healthcare workers (HCWs) is an area of concern because of HCWs' roles as trusted sources of health information, and because of their greater personal exposure to infections acquired in a healthcare setting. This is particularly the case in HCWs of ethnic minorities, who comprise a high proportion of NHS workers in the UK. Data from one NHS trust show lower rates of covid-19 vaccination in ethnic minority HCWs (70.9\% in White workers versus $58.5 \%$ in South Asian and $36.8 \%$ in Black workers; $\mathrm{P}<0.001$ for both). ${ }^{20}$

\section{What are the causes of covid-19 vaccine hesitancy?}

Confidence in the importance of vaccines has the strongest association with vaccine uptake; however, confidence in the importance (necessity and value), safety, and effectiveness of vaccines fell in many countries between 2015 and 2019. ${ }^{21}$ WHO listed 
vaccine hesitancy among the top 10 global threats to health in 2019.22 Drivers of low confidence in covid-19 vaccination are listed in box 1. The 'Understanding Society' UK Household Longitudinal survey highlighted that the main reason for hesitancy was concerns about future unknown effects, with $42.7 \%$ of respondents specifying this. ${ }^{14}$ Less common reasons included those under the bracket of "other" (12.2\%), worries about side effects (11.4\%), concern that others are in more urgent need of the vaccine $(7.7 \%)$, and lack of trust in vaccines (7.6\%). ${ }^{14}$ However, the survey found that people of Black ethnicities were more likely to state that they "don't trust vaccines" compared with White people (29.2\% v 5.7\%), and people of Pakistani and Bangladeshi ethnicities often cited concerns about vaccine side effects $(35.4 \% v 8.6 \%){ }^{14}$ Some reports indicate a rise in vaccine hesitancy following the AstraZeneca vaccine safety scare across Europe and Africa. ${ }^{24} 25$ Historical precedents show that widely publicised safety scares can have profound and long-lasting effects on vaccine confidence. ${ }^{26}$

Box 1: Causes and drivers of low confidence in covid-19 vaccines 5791123

- Socioeconomic and healthcare inequalities and inequities

- Structural racism and previously unethical research involving some ethnic minority groups

- Social disadvantages including lower levels of education and poor access to accurate information

- Misinformation, disinformation, rumours, and conspiracy theories, in particular through social media

- Lack of effective public health messages or targeted campaigns

- Barriers to access, including vaccine delivery time, location, and cost related to socioeconomic inequalities and marginalisation

\section{How to approach covid-19 vaccine hesitancy}

Approaching vaccine hesitancy is complex, and therefore no single intervention can address this entirely, especially in the context of covid-19 where evidence for effective strategies to address it is currently limited. ${ }^{27}$ When considering the most effective methods to increase vaccine uptake, we advocate comprehensive multi-component approaches tailored to the local population, combined with good communication at an individual level. ${ }^{27}$ At a broader national level, a multifaceted, non-stigmatising approach is needed to share communication (in a variety of mediums) from trusted sources. ${ }^{11}$ This includes traditional media channels (for example, television, radio, public transport advertising, and internet) to engage different groups regarding public health policies and counter any misinformation. ${ }^{28} 29$

Recognising barriers to uptake (box 2) helps to inform appropriate interventions to address them (box 3). The key is to build confidence, particularly listening to people's concerns, being respectful of different religious or cultural beliefs, and being aware of historically rooted understandable mistrust, as well as other ethical considerations around clinical interventions. ${ }^{11}$ They will usually be open to engage in dialogue about vaccine safety, efficacy, and importance, and discuss the risks and benefits of vaccination.

Box 2: Stated reasons for low uptake of covid-19 vaccines among the public $^{21} 3031$

- Concerns about long term effects, side effects, and unknown future effects on health

- Previous side effects to other routine vaccines such as influenza vaccine
- Low confidence in vaccines, including their importance, safety, and efficacy

- Lack of trust in the manufacturing and country of production of vaccines, vaccine technology, the pharmaceutical industry, government, and public health bodies

- Concerns about the speed of development of covid-19 vaccines

- Concerns about vaccines' incompatibility with religious beliefs

- Previously negative experiences of healthcare, including racial discrimination

- Lower risk and perception of lower risk of covid-19 (especially among younger age groups)

- Lack of communication from trusted providers and community leaders

- Practical concerns such as inconvenient vaccine delivery time and location

- Not offered vaccine because of inaccurate patient contact information

- Direct and indirect costs of vaccine (in some low and middle income countries)

- Apprehensions surrounding fertility, pregnancy, and breastfeeding

- Belief in conspiracy theories such as covid-19 not being real, or that vaccines modify DNA

- Recent covid-19 infection

Box 3: Summary of strategies for interventions to increase vaccination uptake $^{112732}$

- Offer tailored communication from trusted sources such as community representatives, healthcare providers, and local authorities that is culturally relevant and accessible in multiple languages.

- Improve access to vaccines. This may include flexible delivery models in the community, such as GP practices and outreach programmes with good transportation links.

- . Community engagement. Work with community champions, youth ambassadors, faith leaders, and healthcare workers to raise knowledge and awareness on vaccinations; celebrate household members, friends, relatives, and role models being vaccinated; foster an approach of community immunity and helping others; and create locally developed action plans and a continuous, open, and transparent dialogue.

- Training and education of those involved with engagement activities at a local level: use relevant educational materials (eg, eLearning modules) in presentations and communication skills training.

HCWs are a trusted source of information on vaccination and can influence local vaccination rates in individuals and at a population level. HCWs working alongside local authority members, faith leaders, and "community champions" can facilitate engagement, guide household decision makers, and make vaccine recommendations. ${ }^{1133}$

\section{Improving access and removing barriers}

Historically, interventions based on reminder/recall notifications have improved vaccination uptake in several groups and settings, although limited evidence supports their use specifically for addressing hesitancy. ${ }^{23} 34$

Offering appointments in the evenings or weekends may improve accessibility for some. People with disabilities or those who have been shielding may find it particularly hard to attend a vaccination appointment. Considering an individual's distance from the vaccination site, offering to arrange appropriate transportation, or using home visiting facilities can maximise access for these 
patients. ${ }^{11}$ Vaccination sites away from formal healthcare settings, such as places of worship and work based environments, can offer a degree of familiarity and enable reach within communities that distrust government or medical sources. ${ }^{33}$ They also offer the opportunity for peer support from friends, family members, and colleagues who have agreed to be vaccinated.

\section{Community engagement and local interventions}

Religious or traditional community leaders can engage key audiences through open discussion, advocacy, and integrated community activities. ${ }^{23}$ Where appropriate, this could be alongside group discussions with HCWs in local settings to improve awareness, reinforce messages, and promote consistency. ${ }^{232728}$ Having readily available online material for HCWs and vaccine recipients-such as eLearning modules-will reinforce messaging for vaccine safety and effectiveness. ${ }^{28}$

Consider engaging local health, political, community, legal, and academic representatives to help authorities understand relevant issues and build trust with community partners. ${ }^{35}$ Written, audio, or visual information might be translated into a range of suitable languages or produced in a more accessible format (box 4).3235-38

\section{Box 4: Online resources}

- NHS Choices. Coronavirus vaccine. https://www.nhs.uk/conditions/vaccinations/

- NHS England Coronavirus (covid-19) Resource Centre. https://coronavirusresources.phe.gov.uk/covid-19-vaccine/resources/

- Vaccine Knowledge Project. https://vk.ovg.ox.ac.uk/vk/

- Video by Gavi the Vaccine Alliance. Four types of vaccines and how they work. https://www.youtube.com/watch?v=IFjIVIIcCVc

- WHO Global Vaccine Safety. https://www.who.int/vaccine_safety/en/

- US Centers for Disease Control and Prevention: Vaccines and Immunisations. https://www.cdc.gov/vaccines/covid-19/index.html

- The covid-19 vaccine communication handbook. https://hackmd.io/@scibehC19vax/home

- Johns Hopkins Medicine. https://www.hopkinsmedicine.org/health/conditions-and-diseases/coronavirus/is-thecovid19-vaccine-safe

- British Medical Association. Covid-19: how to communicate with different groups about the vaccine. https://www.bma.org.uk/adviceand-support/covid-19/vaccines/covid-19-how-to-communicate-withdifferent-groups-about-the-vaccine

Regular reporting of vaccine uptake at a local level by different population demographics, including ethnicity, can help to monitor the overall vaccine coverage and identify where resources need to be further targeted. ${ }^{11}$

\section{Discussing vaccination with a patient}

Discussion and engagement with patients who are vaccine hesitant should be conducted in an open, honest, and non-judgmental manner (boxes 5,6 ). ${ }^{39}$ HCWs are well placed to have these conversations given their expertise in communication skills and biomedical training, although evidence is lacking to say whether vaccination uptake is greater if HCWs discuss vaccinations, versus staff in administrative roles. Various approaches have been studied, ${ }^{23}$ from calling patients who have not received a vaccine to supplying online or paper based resources to inform patients of benefits and safety issues. One simple example is the "elicit-share-elicit" approach. ${ }^{32}$ The HCW asks open ended questions to identify concerns and then offers to share their expertise about this concern. Tailored education based on specific attitudes, and previous experiences have also been shown to be beneficial. ${ }^{39}$ This approach maintains an empathetic relationship, and provides an opportunity to communicate risk and support for decision making. For example, if a patient tells you they are concerned about the speed of the vaccine rollout, you might highlight the accelerated collaborative international drive that has taken place, which has occurred without compromising on scientific rigour to establish safety and efficacy including through ethics approval and expert peer review and will be continuously monitored by regulators.

Recent concerns over a possible link between coronavirus vaccines and rare blood clots show that this monitoring is taking place, and can even detect serious side effects as rare as one in 250 ooo people vaccinated..$^{40}$

\author{
- Educational online or written material \\ - Specialist immunisation clinics \\ - Tailored education \\ - "Elicit-share-elicit" approach \\ - Active listening \\ - Motivational interviewing
}

Box 5: Individual level interventions for healthcare workers 39

Box 6: Top tips for HCWs communicating with vaccine-hesitant patients ${ }^{36}$

- Be aware of cultural and emotional differences

- Recognise the unique contexts, such as difficulties in accessing healthcare and adhering to public health guidance

- Provide clear and up-to-date guidance

- Repeatedly check understanding

- Adjust styles for differing literacy, education, and language levels

- Have reliable, up-to-date, and accessible sources of information on hand

- Avoid using jargon and stigmatising language

- Support equity by identifying and targeting vulnerable groups

Exploring the person's priorities-what they have looked forward to, or missed most during the current pandemic-can help to contextualise the importance of the vaccination programme as a collective effort to enable society to come out of lockdown, reverse restrictions, and minimise economic hardships.

One of the key difficulties can be communicating risk, for example, the risk of developing severe infection from covid-19 versus the risk of developing symptoms following vaccination. Understanding a patient's perceptions of risk and health beliefs is key to establishing a shared dialogue through which HCWs can discuss data clearly, using simplified language where appropriate. Engaging with patients on the dynamic, evolving, and at times uncertain nature of scientific evidence is also imperative, especially in the context of covid-19 and vaccines.

\section{Education into practice}

- Do you have a local policy for identifying and engaging patients who are vaccine hesitant in general, and specifically for covid-19 vaccine hesitancy?

- What strategies have you used to increase vaccine uptake in your facility and how have you monitored their impact? 
- What online and community resources do you have access to for increasing vaccine confidence?

\section{How this article was made}

This article uses best available evidence, recent research papers, the latest advice from the World Health Organization, and expert opinion. We searched systematic reviews, other relevant published research, and latest guidelines using MEDLINE, EMBASE, and Google Scholar in March and April 2021. Additional resources were drawn from our personal datasets.

\section{How patients were involved in the creation of this article}

A patient read the manuscript and provided feedback on the relevance and usefulness of the recommendations. The patient specifically requested that we also discuss the root causes of low take up of vaccine among ethnic minorities, including wider determinants of health.

Competing interests: none declared. Further details of The BMJ policy on financial interests are here: https://www.bmj.com/about-bmi/resources-authors/forms-policies-and-checklists/declaration-competinginterests

Provenance and peer review: commissioned, based on an idea from the author; externally peer reviewed.

1 Ritchie H, Ortiz-Ospina E, Beltekian D, et al. The Our World in Data COVID vaccination data. Our World in Data 2021. https://ourworldindata.org/covid-vaccinations

2 World Health Organization. Coronavirus disease (COVID-19): vaccines safety. 2021. https://www.who.int/news-room/q-a-detail/coronavirus-disease-(covid-19)-vaccines-safety

3 Centers for Disease Control and Prevention. Vaccine safety and monitoring. 2021. https://www.cdc.gov/coronavirus/2019-ncov/vaccines/safety.html

4 Majeed A, Molokhia M. Vaccinating the UK against covid-19. BM/2020;371:m4654. doi: 10.1136/bmj.m4654 pmid: 33257346

5 MacDonald NESAGE Working Group on Vaccine Hesitancy. Vaccine hesitancy: Definition, scope and determinants. Vaccine 2015;33:4161-4. doi: 10.1016/i.vaccine.2015.04.036 pmid: 25896383

6 Larson HJ, Jarrett C, Eckersberger E, Smith DM, Paterson P. Understanding vaccine hesitancy around vaccines and vaccination from a global perspective: a systematic review of published literature, 2007-2012. Vaccine 2014;32:2150-9.

doi: 10.1016/j.vaccine.2014.01.081 pmid: 24598724

7 Mills M, Rahal C, Brazel D, et al. Covid-19 vaccine deployment: behaviour, ethics, misinformation and policy strategies. The Royal Society \& The British Academy. 2020. https://royalsociety.org//media/policy/projects/set-c/set-c-vaccine-deployment.pdf

8 Duffy B, Beaver K, Meyer C, et al. Coronavirus: vaccine misinformation and the role of social media. The Policy Institute. 2020. https://www.kcl.ac.uk/policy-institute/assets/coronavirusvaccine-misinformation.pdf

9 Razai MS, Osama T, McKechnie DGJ, Majeed A. Covid-19 vaccine hesitancy among ethnic minority groups. BMJ 2021;372:n513. doi: 10.1136/bmj.n513 pmid: 33637577

10 Razai MS, Kankam HKN, Majeed A, Esmail A, Williams DR. Mitigating ethnic disparities in covid-19 and beyond. BMJ 2021;372:m4921. doi: 10.1136/bmj.m4921 pmid: 33446485

11 UK Government Scientific Advisory Group for Emergencies (SAGE). Factors influencing covid-19 vaccine uptake among minority ethnic groups, 2020. https://www.gov.uk/government/publications/factors-influencing-covid-19-vaccine-uptake-among-minority-ethnic-groups-17-december2020

12 Institute of Global Health Innovation, Imperial College London. Covid-19: Global attitudes towards a COVID-19 vaccine. 2021. https://www.imperial.ac.uk/media/imperial-college/institute-of-globalhealth-innovation/GlobalVaccinelnsights_ICL-Covid-19-Behaviour-Tracker-EMBARGOED-00.0104.02.2021.pdf

13 Robinson E, Jones A, Daly M. International estimates of intended uptake and refusal of COVID-19 vaccines: A rapid systematic review and meta-analysis of large nationally representative samples. medRxiv 2020 [preprint] doi: 10.1101/2020.12.01.20241729

14 Robertson E, Reeve KS, Niedzwiedz CL, etal. Predictors of COVID-19 vaccine hesitancy in the UK household longitudinal study. Brain Behav Immun 2021;94:41-50. doi: 10.1016/j.bbi.2021.03.008 pmid: 33713824

15 Office of National Statistics. Coronavirus and vaccination rates in people aged 70 years and over by socio-demographic characteristic, England: 8 December 2020 to 11 March 2021. 2021. https://www.ons.gov.uk/peoplepopulationandcommunity/healthandsocialcare/healthinequalities/bulletins/coronavirusandvaccinationratesinpeopleaged70yearsandoverbysociodemographiccharacteristicengland/8december2020to11march2021

16 Bambra C, Riordan R, Ford J, Matthews F. The COVID-19 pandemic and health inequalities. J Epidemiol Community Health 2020;74:964-8.pmid: 32535550

17 Mangtani P, Breeze E, Kovats S, Ng ES, Roberts JA, Fletcher A. Inequalities in influenza vaccine uptake among people aged over 74 years in Britain. Prev Med 2005;41:545-53. doi: 10.1016/j.ypmed.2005.02.001 pmid: 15917051
18 Jain A, van Hoek AJ, Boccia D, Thomas SL. Lower vaccine uptake amongst older individuals living alone: a systematic review and meta-analysis of social determinants of vaccine uptake. Vaccine 2017;35:2315-28. doi: 10.1016/j.vaccine.2017.03.013 pmid: 28343775

19 Seasonal influenza vaccine uptake in children of primary school age [collection]: Public Health England. https://www.gov.uk/government/statistics/seasonal-flu-vaccine-uptake-in-children-ofprimary-school-age-monthly-data-2019-to-2020

20 Martin CA, Marshall C, Patel P, et al. Association of demographic and occupational factors with SARS-CoV-2 vaccine uptake in a multi-ethnic UK healthcare workforce: a rapid real-world analysis. medRxiv 2021 [preprint] doi: 10.1101/2021.02.11.21251548

21 de Figueiredo A, Simas C, Karafillakis E, Paterson P, Larson HJ. Mapping global trends in vaccine confidence and investigating barriers to vaccine uptake: a large-scale retrospective temporal modelling study. Lancet 2020;396:898-908. doi: 10.1016/S0140-6736(20)31558-0 pmid: 32919524

22 World Health Organization. Ten threats to global health in 2019. 2019. https://www.who.int/newsroom/spotlight/ten-threats-to-global-health-in-2019

23 World Health Organization. Strategies for addressing vaccine hesitancy - a systematic review: WHO SAGE working group dealing with vaccine hesitancy. 2014. https://www. who.int/immunization/sage/meetings/2014/october/3_SAGE_WG_Strategies_addressing_vaccine_hesitancy_2014.pdf

24 YouGov. Europeans now see AstraZeneca vaccine as unsafe, following blood clots scare. YouGov 2021. https://yougov.co.uk/topics/international/articles-reports/2021/03/22/europeans-now-seeastrazeneca-vaccine-unsafe-follo

25 Dahir AL. Vaccine hesitancy runs high in some African countries, in some cases leaving unused doses to expire. New York Times 2021. https://www.nytimes.com/2021/04/16/world/vaccinehesitancy-africa.html

26 Razai MS, Osama T, Majeed A. Covid-19 vaccine adverse events: balancing monitoring with confidence in vaccines. BMJ Opinion 2021. https://blogs.bmj.com/bmi/2021/03/19/covid-19vaccine-adverse-events-balancing-monitoring-with-confidence-in-vaccines/

27 Jarrett C, Wilson R, O'Leary M, Eckersberger E, Larson HJSAGE Working Group on Vaccine Hesitancy. Strategies for addressing vaccine hesitancy - a systematic review. Vaccine 2015;33:4180-90. doi: 10.1016/j.vaccine.2015.04.040 pmid: 25896377

28 Tull K. K4D Helpdesk Report 672. Vaccine hesitancy: guidance and interventions. Institute of Development Studies. 2014. https:/opendocs.ids.ac.uk/opendocs/handle/20.500.12413/14747

29 Arede M, Bravo-Araya M, Bouchard É, etal. Combating vaccine hesitancy: teaching the next generation to navigate through the post truth era. Front Public Health 2019;6:381. doi: 10.3389/fpubh.2018.00381 pmid: 30693276

30 Robertson E, Reeve KS, Niedzwiedz CL, et al. Predictors of COVID-19 vaccine hesitancy in the UK Household Longitudinal Study. medRxiv 2021 [preprint] doi: 10.1101/2020.12.27.20248899

31 Office for National Statistics. Coronavirus and the social impacts on Great Britain: 29 January 2021. 2021. https://www.ons.gov.uk/peoplepopulationandcommunity/healthandsocialcare/healthandwellbeing/bulletins/coronavirusandthesocialimpactsongreatbritain/29january2021

32 Lewandowsky S, Cook J, Schmid P, et al. The COVID-19 Vaccine Communication Handbook. A practical guide for improving vaccine communication and fighting misinformation: SciBeh, 2021

33 Peterson P, McNabb P, Maddali SR, Heath J, Santibañez S. Engaging communities to reach immigrant and minority populations: the Minnesota Immunization Networking Initiative (MINI), 2006-2017. Public Health Rep 2019;134:241-8. do: 10.1177/0033354919834579 pmid: 30912998

34 Williams N, Woodward H, Majeed A, Saxena S. Primary care strategies to improve childhood immunisation uptake in developed countries: systematic review. JRSM Short Rep 2011;2:81. doi: 10.1258/shorts.2011.011112 pmid: 22046500

35 UK Government. Public health messaging for communities from different cultural backgrounds: Scientific Pandemic Influenza Group on Behaviours (SPI-B), 2020. https://www.gov.uk/government/publications/spi-b-consensus-on-bame-communication-22-july-2020

36 British Medical Association. COVID-19: how to communicate with different groups about the vaccine. 2021. https://www.bma.org.uk/advice-and-support/covid-19/vaccines/covid-19-how-tocommunicate-with-different-groups-about-the-vaccine

37 UK Government. UK COVID-19 vaccine uptake plan. 2021. https://www.gov.uk/government/publications/covid-19-vaccination-uptake-plan/uk-covid-19-vaccine-uptake-plan

38 NHS England. COVID-19 vaccine programme. Maximising vaccine uptake in underserved communities: a framework for systems, sites and local authorities leading vaccination delivery, 2021. https://www.england.nhs.uk/coronavirus/publication/maximising-vaccine-uptake-in-underserved-communities-a-framework/

39 European Centre for Disease Prevention and Control. Catalogue of interventions addressing vaccine hesitancy. 2017. https://www.ecdc.europa.eu/en/publications-data/catalogue-interventionsaddressing-vaccine-hesitancy

40 Public Health England. COVID-19: the green book, chapter 14a. 2021. https://assets.publishing.service.gov.uk/government/uploads/system/uploads/attachment_data/file/978508/Green_book_chapter_16April2021.pdf

This article is made freely available for use in accordance with BMJ's website terms and conditions for the duration of the covid-19 pandemic or until otherwise determined by BMJ. You may use, download and print the article for any lawful, non-commercial purpose (including text and data mining) provided that all copyright notices and trade marks are retained. 